\title{
Proceedings of the summer meeting of the British Neuropsychiatric Association, Cambridge, 14-16 July 1996
}

The topics of the meeting were neurodevelopment and language.

Professor D Skuse (London) was the keynote speaker in the neurodevelopment session with his talk on neurodevelopmental changes in childhood: illuminating brain-behaviour relationships. Corticogenesis involves a number of interlinked processes (cell migration, remodelling, and pruning of axons and synapses) which start in utero and continue postnatally. Abnormalities at various stages of corticogenesis can lead to mental retardation, epilepsy, and focal developmental disorders. This continuing process of maturation is reflected in the changing pattern of cognitive abilities that can be elicited at different developmental stages (prenatal recognition of mother's voice and the neonate's ability to recognise facelike objects). The development of executive functions takes place around the age of 3 to 4 years and is a key step in cognitive maturation. A well known example of executive function is the acquisition of the "theory of mind", an ability to infer other people's mental states, which involves planning and the use of inner language and deception. Another important aspect of cognitive development is the appearance of gender related differences. Some of these can be demonstrated in adults using functional imaging. For instance, unilateral activation with verbal reasoning is seen in males, whereas bilateral activation patterns are commoner in females, although considerable variability between the sexes is present. Professor Skuse suggested that Turner's syndrome is a useful model to study gender related cognitive impairment and to elucidate the molecular genetic basis of cognition.

Professor A Copp (London) discussed the genetic basis of early nervous system development and its disorders. The main focus of genetic research is to identify genes responsible for disease entities using genetic linkage analysis to locate and clone the critical gene. Genetic targeting, a complementary research strategy in mice, provides the opportunity to study the neurobehavioural phenotypes which result when genes relevant to the development of the nervous system are inactivated. Professor Copp presented his own research using a mouse genetic model of spina bifida. By focusing on the interactions that mediate the effect of disease genes, it has been possible to determine the extensive sequence of molecular, cellular, and morphogenic events underlying the genetic defect. It has also been possible to develop strategies (supplementation with the vitamin myo-inositol) potentially useful to prevent these defects in humans.

Dr L Rifkin (London) presented his study of very preterm infants grown-up: the frequency and significance of structural brain abnormalities at 14 years of age. Very preterm infants ( $<33$ weeks gestation) and low birth weight infants $(<1500 \mathrm{~g})$ have an increased risk of brain injury and show a range of neurological, behavioural, and cognitive abnormalities in childhood. A cohort of these infants were examined at 14 years of age to determine the frequency of MRI brain abnormalities and their clinical relevance. Compared with an age matched control group, preterm infants had a significant increase in MRI, neurological, and cognitive abnormalities when grown up and tended to have more behavioural disturbances. However, MRI abnormalities were poorly correlated with other outcome measures. On the other hand, neurological abnormalities at 14 were good predictors of cognitive function and school performance.

Dr L Wing (London) discussed movement problems and social impairments in autistic spectrum disorders. Autistic spectrum disorders include typical autism, Asperger's syndrome, and patients described as "loners" by Wolff. Impairment of social interaction, communication, and imagination together with a narrow, repetitive pattern of interests and activities characterise the disorder. In addition, odd responses to sensory stimuli, unusual sleeping, eating, and drinking patterns, abnormalities of posture and movements, motor and vocal stereotypies and tics, and bizarre behaviour are commonly seen. These motor abnormalities may become more severe with age leading in some patients to the additional diagnoses of Tourette's syndrome, obsessive-compulsive disorder, and catatonia. These movement abnormalities are not mutually exclusive and the same patient may exhibit them at different times. Equally intriguing is the fact that features of the autistic spectrum can also be found in patients with Tourette's syndrome, obsessive-compulsive disorder, or as part of the sequelae of encephalitis lethargica. The frequency of these associations remains to be determined. Comparative neuropathological studies are eagerly awaited.

The following short papers were presented: Accounting for the relationship between language, literacy, and phonological awareness in terms of shared genetic influences ( $\mathrm{Dr} B$ Hohnen); Misdiagnosis in conversion disorder (Dr H Crimlisk); An extraordinary case of somatisation disorder: Baron Munchausen Mark II (Dr J Bird); Hyperphagic short stature: a genetic syndrome? (Dr J Gilmour); Is there a link between temporal lobe symptoms and affective disorder in migraine? (Dr E McEvedy); Two cases of central pontine myelinolysis (Dr D Fitzpatrick); Neuropsychiatric sequelae of palidotomy for Parkinson's disease: a pilot study (Dr $M$ Lambert); Depression, attentional bias, and anterior cingulotomy ( $\mathrm{Dr} \mathrm{H}$ Ring); Neurological aspects of schizophrenia: a family study (Dr T Griffiths)

The keynote speaker in the language session was Professor R Plomin (London), who discussed the genetic and developmental origins of cognitive and language development in children. Work done on the heritability of various diseases using twin studies has shown that disorders such as autism and idiopathic epilepsy have a higher genetic component than previously thought, whereas others, in whom the genetic component was thought to be significant - for example, alcoholism and Parkinson's disease-have lower heritability. What these studies all demonstrate, however, is the synergistic relation that exists between genetic and environmental aetiologies. 
Professor Plomin explained how, with data from a large twin register, it would be possible to extend heritability studies to investigate how genes may drive the environment and the mechanisms by which genes act, enabling the bringing together of the disciplines of quantitative genetics and molecular genetics in the investigation of qualitative trait loci for complex behavioural traits. The recently created research centre at the Institute of Psychiatry, by bringing together these disciplines, will make possible this type of study.

Dr R Wise (London) gave a paper entitled input, output, and time in which he discussed some of the problems and limitations of functional imaging. Substraction methodology has commonly been used in activation experiments on the assumption that the behavioural differences between task and control conditions are confined to those under investigation. This method, akin to "cognitive phrenology", works well for the exploration of motor and visual systems. Two alternative approaches may prove more useful to study cognitive deficits. The first relies on the effect of implicit processing of meaningful stimuli (for example, the simple listening to a word results in negative correlation of imageability and activation in the left superior temporal sulcus). An example of the second experimental design is the study of the regions involved in speech perception using distorted consonant/vowel combinations in six steps. The variable familiarity of the stimulus allows the study of a very distributed system involved in many aspects of the task (perception, response, and learning). These new techniques are particularly useful when applied to patients unable to perform explicit tasks with the speed and accuracy of controls and demonstrate the impact of focal pathology on parallel distributed neural systems.

Dr A Thacker (London) described her work on the language disorder in deaf patients with schizophrenia. Using transcriptions of the language of prelingually deaf subjects, who communicated predominantly by British Sign Language (BSL), she compared the errors made by schizophrenic deaf subjects and normal deaf controls. Potential confounders are the variable skill in the use of BSL and its problematic syntax. There were no significant differences between the groups in the age of acquisition of BSL, but schizophrenic subjects made errors such as incoherence, derailment, perseveration, illogicality, and paraphasias which seem to be linked to the presence of psychopathology. Further research is needed to confirm these findings and to explore their relation to other cognitive deficits such as action planning.

The topic of Professor Christine Temple's presentation was the language ability and disability in Turner's syndrome. Girls with Turner's syndrome provide an opportunity for examining the effect of genetic composition (in this case the presence of only one $\mathrm{X}$ chromosome, or an X chromosome deletion) and its environmental consequences (intrauterine growth in the absence of normal exposure to endogenous sex hormones) on the behavioural phenotype. Professor Temple mentioned that, by contrast with the well described visuospatial deficits, girls with Turner's syndrome have significantly enhanced verbal skills, including superior recognition memory for words, hyperlexia, better vocabulary, and comprehension skills, when allowances are made for IQ, age, and "normal" male/female differences. She went on to discuss some of the reasons why this apparent superiority may have been overlooked in previous studies, which often relied on verbal skills such as fluency.

The afternoon session was opened by Dr Tim Crow who discussed the implications of hemispheric indecision on the evolution of language. Using data from the UK National Child Development Survey, he presented findings suggesting that academic ability was related to a continuous handedness variable. Strong right handers were modestly impaired in both verbal and nonverbal ability; however, the greatest impairments were found at the point of equal hand skill or the point of "hemispheric indecision". Preschizophrenic children were found to be closer to this point than others in the cohort. Dr Crow proposed that the findings added weight to the hypothesis that a dimension related to cerebral dominance could account for major variance in cognitive and language skills as well as psychopathology. He described and discussed the relevance of the "right shift factor", as described by Annett, proposing that a single gene or several genes, under continual evolutionary selection, could account for the biological disadvantage of hemispheric indecision, and went on to discuss the evidence for the occurrence of a handedness gene in the XY homologous genes.

The session ended with the following scientific papers and video case presentations: Conversational discourse in Alzheimer's disease and frontotemporal dementia ( $\mathrm{Ms} \mathrm{H}$ Griffiths); The interrelation between classic motor neuron disease and frontotemporal dementia: a neuropsychological and ${ }^{99}$ Tc-HMPAO SPECT study (Dr $P$ Talbot: winner of the 1995 BNPA prize); SPECT and Lewy body disease (Dr A Varma); Changes in pupillary diameter and the kinetics of the darkness and light reflexes in old age and Alzheimer's disease (Professor E Szabadi).

At the Annual General Meeting Dr John Hodges, consultant neurologist and lecturer (Cambridge), was elected to replace Professor David Neary as a committee member. The BNPA prize was awarded to Dr Alexander J Mitchell for his essay entitled the neurobiological correlates of poststroke major depression: overview and implications for primary depressive disorder.

The 1997 winter meeting of the BNPA will take place in London on Friday 17 January at London Zoo (Regent's Park) and the topic for the meeting will be memory.

M A RON H CRIMLISK

The Institute of Neurology and The National Hospital for Neurology and Neurosurgery Queen Square, London WC1N 3BG, UK 